\title{
Some generalizations in the mathematical developments of the theory of the geodetic overdetermined problems
}

\author{
F. SACERDOTE and F. SANSÒ
}

\begin{abstract}
Two particular cases of the overdetermined gravimetry-gradiometry problem are discussed:

a) the case of a latitude-dependent statistical weight for gradiometric data, corresponding to a data distribution coming from satellite polar orbits,

b) the case of a volume distribution, instead of a surface distribution, for satellite gradiometric data.

In both cases a discussion of numerical methods for solving the problem with realistic data is started; for case b) an analytic solution is found under simplifying assumptions.
\end{abstract}

\section{INTRODUCTION}

The theory of overdetermined boundary-value problems in physical geodesy has been developed in order to handle with rigorous mathematical procedures situations in which different kinds of data are available from satellite and ground measurements, and are used as boundary conditions to solve the boundary-value problem for the earth's potential.

Usually the different data sets are inconsistent owing to measurement errors and a abest) estimate of the solution is looked for, according to some minimum principle arising from the stochastic structure of the errors.

A general formulation is given by Sanso (1988), who describes the stochastic properties of the boundary data by introducing Wiener measures on the boundary surface. A different starting point is assumed by Rummel and Teunissen (1987), who consider the problems with data continuously distributed over the whole surface as limit cases of situations in which a growing number of discrete data is given and a finite set of parameters is determined.

1980 Mathematics Subject Classification (1985 revision): 35 R99.

Editorial de la Universidad Complutense. Madrid, 1990. 
Practical examples are discussed by Sacerdote and Sansò (1985a, b, 1987); in particular, some simple cases of the gravimetry-gradiometry problem have been treated in this theoretical frame, and some results have been obtained, concerning the relative influence of gravity and gravity gradient data in the determination of different harmonic components of the earth's potential.

Yet, analytical solutions can be obtained only under drastically simplifying assumptions. First, only the components of the gravity gradient tensor containing radial derivatives are introduced as data. Second, the different kinds of data - even the different components of the gravity gradient - are assumed to be uncorrelated, and the statistical weights are supposed to be constant all over the surface. Finally, the data are given on spherical surfaces not necessarily on the boundary surface. To take into account the case of satellite measurements, buy anyway on a surface. A more realistic approach would require that satellite data be distributed in a shell between the minimum and the maximum heights of elliptic orbits.

The present paper deals with computational difficulties that arise when some of these simplifying assumptions are dropped. More precisely, in section 2 the case of a statistical weight depending on the latitude is investigated; in section 3 the problem is reformulated for data distributed on a spherical shell.

\section{NON-CONSTANT STATISTICAL WEIGHTS}

First, the main features of the mathematical developments discussed in previous papers (Sacerdote \& Sansò 1987), (Sansò 1988) are briefly summarized.

Assume for simplicity that the data sets are two different scalar quantities: one measured at ground level (for example, gravity anomaly), one got out from satellite measurements (for example, the double radial derivative of the anomalous potential). In addition, the earth's surface is conventionally represented by a spherical surface $S_{1}$ with radius $R_{1}$, and the satellite orbits are supposed to lie on a concentric spherical surface $S_{2}$ with radius $R_{2}>R_{1}$.

Hence the problem is formulated as follows

$$
\left\{\begin{array}{l}
\nabla^{2} T=0 \\
B_{1} T=f_{1}^{(0)} \\
B_{2} T=f_{2}^{(0)}
\end{array}\right.
$$

where $B_{1}$ and $B_{2}$ are operators that characterize the "boundary" condition on $S_{1}$ and $S_{2}$ respectively. The data are represented by surface stochastic meas- 
ures, that can be split into a deterministic part and a zero-mean Wiener measure:

$$
\begin{aligned}
& \mu_{1}^{(0)}\left(d S_{1 Q}\right)=B_{1} T(Q) d S_{1 Q}+\mu_{1}^{W}\left(d S_{1 Q}\right) \\
& \mu_{2}^{(0)}\left(d S_{2 Q}\right)=B_{2} T(Q) d S_{2 Q}+\mu_{2}^{W}\left(d S_{2 Q}\right)
\end{aligned}
$$

$d S_{1 Q}, d S_{2 Q}$ are the usual Lebesgue measures on $S_{1}$ and $S_{2}$; the Wiener measures $\mu_{1}^{W}, \mu_{2}^{W}$ are families of normal variables, indexed by subsets of $S_{1}$ and $S_{2}$ respectively, with covariances

$$
\begin{aligned}
& E\left\{\mu_{1}^{W}(A) \mu_{1}^{W}\left(A^{\prime}\right)\right\}=\int_{A \cap A^{\prime}} p_{1}^{-1}(Q) d S_{1 Q} \\
& E\left\{\mu_{2}^{W}(D) \mu_{2}^{W}\left(D^{\prime}\right)\right\}=\int_{D \cap D^{\prime}} p_{2}^{-1}(Q) d S_{2 Q} \\
& E\left\{\mu_{1}^{W}(A) \mu_{2}^{W}\left(D^{\prime}\right)\right\}=0
\end{aligned}
$$

where $p_{1}(Q), p_{2}(Q)$ are suitable weight functions.

Expressions [2.3] generalize formulas [2.4] of (Sacerdote \& Sansò 1987) to the case of non-constant statistical weights. In this frame, following (Sacerdote \& Sansò 1987), the solution of problem [2.1] has the form of a linear estimate in terms of the observables:

$$
\hat{T}(P)=\int_{S_{\mathrm{t}}} g_{1}(P, Q) \mu_{1}^{(0)}\left(d S_{1 Q}\right)+\int_{S_{2}} g_{2}\left(P, Q^{\prime}\right) \mu_{2}^{(0)}\left(d S_{2 Q}\right)
$$

$g_{1}(P, Q)$ e $g_{2}(P, Q)$ have to be determined from the minimization of the quadratic error function

$$
\begin{gathered}
\eta^{2}(P)=E\left\{(\hat{T}(P)-\bar{T}(P))^{2}\right\}=\int_{S_{1}} p_{1}^{-1}(Q) g_{1}^{2}(P, Q) d S_{1 Q}+ \\
+\int_{S_{2}} p_{2}^{-1}\left(Q^{\prime}\right) g_{2}^{2}\left(P, Q^{\prime}\right) d S_{2 Q}
\end{gathered}
$$

under the «unbiasedness» condition

$$
\hat{T}(P)=\int_{S_{1}} g_{1}(P, Q) B_{1} \hat{T}(Q) d S_{1 Q}+\int_{S_{2}} g_{2}\left(P, Q^{\prime}\right) B_{2} \hat{T}\left(Q^{\prime}\right) d S_{2 Q^{\prime}}
$$

In (Sacerdote \& Sansò 1987) condition [2.6] was taken into account by introducing a Lagrange multiplier; in the present case this procedure leads to computational difficulties in the evaluation of the multiplier itself, which is preliminary to the determination of $g_{1}$ and $g_{2}$. Hence a more direct procedure is adopted, which does not enable to get rid of all the difficulties, but at least sheds light upon the expressions for $g_{1}$ and $g_{2}$. 
Assume for example that the boundary-value problem with the boundary condition $B_{1}$ be uniquely solvable and that the solution be expressed by applying an integral operator to the boundary known function:

$$
T(P)=\int_{S_{1}} R(P, Q) f(Q) d S_{1} .
$$

In this case $g_{1}(P, Q)$ can be expressed in terms of $g_{2}(P, Q)$ from condition $[2.6]$. Introducing the Poisson integral kernel $G\left(Q^{\prime}, Q\right),[2.6]$ can be written in the form

$$
\begin{aligned}
& \int_{S_{1}} g_{1}(P, Q) B_{1} T(Q) d S_{1 Q}+\int_{S_{2}} d S_{2 Q^{\prime}} g_{2}\left(P, Q^{\prime}\right) B_{2}\left(\int_{S_{1}} G\left(Q^{\prime}, Q\right) T(Q) d S_{1 Q}\right)= \\
& \quad=\int_{S_{1}} G(P, Q) T(Q) d S_{1 Q}
\end{aligned}
$$

Assume now that $B_{1}$ and $B_{2}$ be self-adjoint with respect to the scalar products on $S_{1}$ and $S_{2}$ respectively (which is certainly true if spherical harmonics are eigenfunctions of $B_{1}$ and $B_{2}$ ); then [2.8] can be written as

$\int_{S_{1}} d S_{1 Q}\left(B_{1 Q} g_{1}(P, Q)+\int_{S_{2}} d S_{2 Q^{\prime}}\left(B_{2 Q^{\prime}} g_{2}\left(P, Q^{\prime}\right)\right) G\left(Q^{\prime}, Q\right)-G(P, Q) T(Q)=0\right.$

that yields, owing to the arbitrariness of $T(Q)$,

$$
B_{1} g_{1}(P, Q)+\int_{S_{2}} d S_{2 Q^{\prime}}\left(B_{2 Q^{\prime}} g_{2}\left(P, Q^{\prime}\right)\right) G\left(Q^{\prime}, Q\right)=G(P, Q)
$$

Finally, by virtue of [2.7], one obtains

$g_{1}(P, Q)=\int_{S_{1}} d S_{1 Q^{\prime \prime}} R\left(Q, Q^{\prime \prime}\right)\left[-\int_{S_{2}} d S_{2 Q^{\prime}} B_{2 Q^{\prime}} g_{2}\left(P, Q^{\prime}\right) G\left(Q^{\prime}, Q^{\prime \prime}\right)+G\left(P, Q^{\prime \prime}\right)\right]$

Now, $g_{1}$ in formula [2.5] has to be replaced by its expression [2.11]. The result can be expressed in the form

$$
\begin{gathered}
\eta^{2}(P)=\int_{S_{2}} d S_{2 Q} \int_{S_{2}} d S_{2 Q^{\prime}} g_{2}(P, Q) g_{2}\left(P, Q^{\prime}\right) K\left(Q, Q^{\prime}\right)-2 \int_{S_{2}} d S_{2 Q} g_{2}(P, Q) \\
\cdot h(P, Q)+\int_{S_{2}} d S_{2 Q} P_{2}^{-1}(Q) g_{2}^{2}(P, Q)+F(P)
\end{gathered}
$$

where

$$
\begin{aligned}
& K\left(Q_{1}, Q_{2}\right)= \\
& \int_{S_{1}} d S_{1 Q} \int_{S_{1}} d S_{1 Q^{\prime}} \int_{S_{1}} d S_{1 Q^{\prime \prime}} p_{1}^{-1}(Q) R\left(Q, Q^{\prime}\right) R\left(Q, Q^{\prime \prime}\right) B_{2 Q_{1}} G\left(Q_{1}, Q^{\prime}\right) \\
& \cdot B_{2 Q_{2}} G\left(Q_{2}, Q^{\prime \prime}\right)=\int_{S_{1}} d S_{1 Q} p_{1}^{-1}(Q) B_{2 Q_{1}} H\left(Q, Q_{1}\right) B_{2 Q_{2}} H\left(Q, Q_{2}\right)
\end{aligned}
$$


$\left(H\left(Q, Q_{i}\right)=\int_{S_{1}} d S_{1 Q} R\left(Q, Q^{\prime}\right) G\left(Q_{j} Q^{\prime}\right)\right) ;$

$$
\begin{aligned}
& h\left(P, Q_{1}\right)=\int_{S_{1}} d S_{1 Q} \int_{S_{1}} d S_{1 Q^{\prime}} \int_{S_{1}} d S_{1 Q^{\prime \prime}} P_{1}^{-1}(Q) R\left(Q, Q^{\prime}\right) R\left(Q, Q^{\prime \prime}\right) G\left(P, Q^{\prime \prime}\right) \text {. } \\
& \cdot B_{2 Q_{1}} G\left(Q_{1}, Q^{\prime \prime}\right)=\int_{S_{1}} d S_{1 Q} p_{1}^{-1}(Q) H(Q, P) B_{2 Q_{1}} H\left(Q, Q_{1}\right) \text {. }
\end{aligned}
$$$$
F(P)=\int_{S_{1}} d S_{1 Q} \int_{S_{1}} d S_{\mathrm{t} Q^{\prime}} \int_{S_{1}} d S_{\mathrm{t} Q^{\prime \prime}} p_{1}^{-1}(Q) R\left(Q, Q^{\prime}\right) R\left(Q, Q^{\prime \prime}\right) G\left(P, Q^{\prime}\right) \text {. }
$$$$
G\left(P, Q^{\prime \prime}\right)=\int_{S_{1}} d S_{1 Q} p_{1}^{-1}(Q) H^{2}(P, Q)
$$

The minimization of $\eta^{2}(P)$ with respect to the variable $g_{2}(P, Q)$ leads to the integral equation

$$
p_{2}^{-1}(Q) g_{2}(P, Q)+\int_{S_{2}} d S_{2 Q^{\prime}} K\left(Q^{\prime}, Q\right) g_{2}\left(P, Q^{\prime}\right)=h(P, Q), Q \in S_{2}
$$

In order to carry on these computations and to clarify the meaning of the formulas obtained up to now, developments into spherical harmonics can be used. The following expressions for the operators $B_{1}$ and $B_{2}$ are introduced:

$$
\begin{aligned}
& B_{1}\left[\left(\frac{R_{1}}{r_{P}}\right)^{l+1} Y_{l m}\left(\sigma_{P}\right)\right]=A_{l} Y_{l m}\left(\sigma_{P}\right) \\
& B_{2}\left[\left(\frac{R_{1}}{r_{P}}\right)^{l+1} Y_{l m}\left(\sigma_{P}\right)\right]=C_{l} Y_{l m}\left(\sigma_{P}\right)
\end{aligned}
$$

Consequently

$$
R(P, Q)=\frac{1}{4 \pi} \sum_{l} A_{l}^{-1}(2 l+1)\left(\frac{R_{1}}{r_{P}}\right)^{l+1} P_{l}\left(\cos \psi_{P_{Q}}\right), Q \in S_{1}
$$

Moreover

$$
G(P, Q)=\frac{1}{4 \pi} \sum_{l}(2 l+1)\left(\frac{R_{1}}{r_{P}}\right)^{l+1} P_{l}\left(\cos \psi_{P Q}\right), Q \in S_{1}
$$

The integrals in [2.13] and [2.14] can be easily computed only under the additional assumption that the weight $p_{1}$ is constant. In this case only the weight $p_{2}$ is allowed to vary with the measurement point. Anyway, even this simplified situation is interesting from the point of view of the geodetic applications, as $p_{2}$ is referred to satellite measurements and can be modelled 
from the distribution of the orbits. More precisely, in the case of polar orbits, which are closer near the poles, a reasonable assumption for $p_{2}(Q)$ is

$$
p_{2}(Q)=\frac{c}{\sin \theta_{Q}}
$$

corresponding to a statistical weight proportional to the number of data per unit area.

Introducing [2.17], [2.18], [2.19], the kernels defined by [2.13], [2.14] can be written as

$$
\begin{gathered}
K\left(Q_{1}, Q_{2}\right)=\frac{p_{1}^{-1}}{4 \pi} \sum_{l, m}\left(\frac{C_{l}}{A_{l}}\right)^{2} Y_{l m}\left(\sigma_{Q_{1}}\right) Y_{l m}\left(\sigma_{Q_{2}}\right), Q_{1}, Q_{2} \in S_{2} \\
h\left(P, Q_{1}\right)=\frac{p_{1}^{-1}}{4 \pi} \sum_{l, m}\left(\frac{C_{l}}{A_{l}^{2}}\right)\left(\frac{R_{1}}{r_{P}}\right)^{l+1} Y_{l m}\left(\sigma_{P}\right) Y_{l m}\left(\sigma_{Q_{l}}\right), Q_{1} \in S_{2}
\end{gathered}
$$
form

As for $g_{2}(P, Q)$, its development into spherical harmonics has the general

$$
g_{2}(P, Q)=\sum g_{l m l^{\prime} m^{\prime}} Y_{l m}\left(\sigma_{P}\right)\left(\frac{R_{1}}{r_{P}}\right)^{l+1} Y_{l^{\prime} m^{\prime}}\left(\sigma_{Q}\right), Q \in S_{2}
$$

Only in the case of constant $p_{2}$ the assumption $l=l^{\prime}, m=m^{\prime}$ can be done, and the result agrees with the formulas obtained in (Sacerdote \& Sansò 1987); in the present case the expression [2.22] must be taken as starting point. Hence the first term in equation [2.16] becomes

$$
\begin{gathered}
P_{2}^{-1}(Q) g_{2}(P, Q)=c^{-1} \sum g_{l m^{\prime} m^{\prime}} Y_{l m}\left(\sigma_{P}\right)\left(\frac{R_{1}}{r_{P}}\right)^{++1} \sin \theta_{Q} \bar{P}_{l^{\prime} m^{\prime}}\left(\cos \theta_{Q}\right) \\
\cdot \begin{cases}\cos m^{\prime} \lambda_{Q} & \text { if } m^{\prime} \geq 0 \\
\sin \left|m^{\prime}\right| \lambda_{Q} & \text { if } m^{\prime}<0\end{cases}
\end{gathered}
$$

and can be handled using the formula

$$
\sin \theta P_{l m}(\cos \theta)=\frac{1}{2 l+1}\left(P_{l+\mathrm{l}, m+1}(\cos \theta)-P_{l-1, m+1}(\cos \theta)\right)
$$

(cf. (Magnus et al. 1966), (4.3.3)). 
It is remarkable that, under the assumption [2.20], as the non-constant statistical weight does not affect the dependence on $\lambda_{Q}$, the expression [2.22] can be simplified by assuming

$$
\begin{aligned}
& g_{l m^{\prime} m^{\prime}}= \begin{cases}0 & m \neq m^{\prime} \\
g_{l l^{\prime} m} & m=m^{\prime}\end{cases} \\
& g_{l r^{\prime} m}=g_{i r,-m}
\end{aligned}
$$

then, equation [2.16] depends actually on the difference $\lambda_{P}-\lambda_{Q}$ and, taking into account [2.24], can be written in the form

$$
\begin{aligned}
& \sum_{l, m \geq 0} \bar{P}_{l m}\left(\cos \theta_{P}\right)\left(\frac{R_{1}}{r_{P}}\right)^{l+1} \cos m\left(\lambda_{P}-\lambda_{Q}\right) \\
& \cdot\left[\sum _ { l \geq m } g _ { l l _ { m } } \left(\frac{c^{-1}}{2 l+1}\left(\bar{P}_{l^{\prime}+1, m+1}\left(\cos \theta_{Q}\right)-\bar{P}_{l^{\prime}-1, m+1}\left(\cos \theta_{Q}\right)\right)+p_{1}^{-1}\left(\frac{C_{l^{\prime}}}{A_{l^{\prime}}}\right)^{2}\right.\right. \\
& \left.\left.\cdot R_{2}^{2} \bar{P}_{l m}\left(\cos \theta_{Q}\right)\right)-\frac{p_{1}^{-1}}{4 \pi} \frac{C_{l}}{A_{l}^{2}} \bar{P}_{l m}\left(\cos \theta_{Q}\right)\right]=0
\end{aligned}
$$

The expression between square brackets must vanish for each fixed $l, m$; yet, it leads to an infinite-dimensional linear system in the unknowns $g_{l r} m$, that can be treated numerically, truncating at a suitable $l^{\prime}=L$. The essential point is that the coefficients of $g_{I^{\prime} m}$ do not depend on $l$, that reduces drastically the amount of computations. [2.26] is transformed into a linear algebraic system by multiplying by $\bar{P}_{j m}\left(\cos \theta_{Q}\right) \sin \theta_{Q}, j=m, \ldots, L$ and integrating over $\theta_{Q}$. The integrals

$$
\int_{0}^{\pi} \bar{P}_{l m}(\cos \theta) \bar{P}_{j k}(\cos \theta) \sin \theta d \theta
$$

can be computed by iteration formulas (Mainville, 1986).

The particular case of gravimetry-gradiometry corresponds to the choice

$$
\begin{array}{ll}
A_{l}=\frac{l-1}{R_{\mathrm{I}}} & \text { (gravity anomaly) } \\
C_{l}=\frac{(l+1)(l+2)}{R_{1}^{2}}-\left(\frac{R_{1}}{R_{2}}\right)^{l+3} \quad \text { (radial gravity gradient) }
\end{array}
$$

It must be recalled that the problem with gravity anomalies as boundary values is not uniquely solvable, unless it is suitably modified; correspondingly, 
in the integral kernel $R(P, Q)$ expressed by [2.18], that in this case is the Stokes kernel, the component of degreee $l=1$ is missing. On the other hand, the $l=1$ component of the potential is not affected by gravity anomalies and is uniquely determined by the corresponding component of the radial gravity gradient. Therefore the solution is split into two parts: a deterministic one, which contains only the $l=1$ component, and another one, containing atl the remaining components, which is obtained with the method previously described and is affected only by the components of the data functions with $l \neq 1$. It has to be pointed out that gravity anomalies should not contain $l=1$ ccomponents, but for measurement errors.

\section{DATA DISTRIBUTED IN A SPHERICAL SHELL}

As pointed out in the introduction, satellite measurements are not usually distributed on a surface, but rather in a thin shell. Hence such data are not suitably described by a stochastic surface measure. Yet, it is not difficult to generalize the computation procedure previously developed by introducing a stochastic volume measure. Namely, in equation [2.2] $\mu_{2}^{(0)}\left(d S_{2 Q}\right)$ is replaced by

$$
\mu_{2}^{(0)}(d Q)=B_{2} T(Q) d Q+\mu_{2}^{W}(d Q)
$$

where $\mu_{2}^{W}$ is a zero-mean Wiener measure, with covariance

$$
E\left\{\mu_{2}^{W}(V) \mu_{2}^{W}\left(V^{\prime}\right)\right\}=\int_{V \cap V^{\prime}} p_{2}^{-1}(Q) d Q
$$

The estimated solution now has the form

$$
\hat{T}(P)=\int_{S_{1}} g_{1}(P, Q) \mu_{\mathrm{l}}^{(0)}\left(d S_{\mathrm{t} Q}\right)+\int_{V} g_{2}\left(P, Q^{\prime}\right) \mu_{2}^{(0)}\left(d Q^{\prime}\right)
$$

where $V$ is the volume in which the data are distributed, namely the shell between two concentric spherical surfaces with radii $R$ and $R+\delta R$ $(\delta R<<R)$.

The quadratic error function to minimize is

$$
\eta^{2}(P)=\int_{S_{1}} p_{1}^{-1} g_{1}^{2}(P, Q) d S_{Q}+\int_{V} p_{2}^{-1} g_{2}^{2}\left(P, Q^{\prime}\right) d Q^{\prime}
$$

and the unbiasedness condition is expressed by

$$
\hat{T}(P)=\int_{S_{1}} g_{1}(P, Q) B_{1} \hat{T}(Q) d S_{Q}+\int_{V} g_{2}(P, Q) B_{2} \hat{T}(Q) d Q^{\prime}
$$


The formal developments of the previous section can be carried out in this case exactly in the same way, simply replacing the integrals over the surface $S_{2}$ with integrals over the volume $V$. The result is the integral equation

$$
p_{2}^{-1}(Q) g_{2}(P, Q)+\int_{V} d Q^{\prime} K\left(Q^{\prime}, Q\right) g_{2}\left(P, Q^{\prime}\right)=h(P, Q), Q \in V
$$

with $K(Q, Q)$ and $h(P, Q)$ as in [2.21]; it can be investigated, as in the previous section, using developments into spherical harmonics. As an example, computations are carried out in the particular case of gravimetry-gradiometry, where the data are expressed as in [2.17], with the specifications given in [2.27]; the only difference is that in the radial gravity gradient the constant radius $R_{2}$ is replaced by the variable $r$. The general development of $g_{2}(P, Q)$ is written in the form

$$
g_{2}(P, Q)=\sum_{l, m}\left(\frac{R_{1}}{r_{P}}\right)^{l+1} Y_{l m}\left(\sigma_{P}\right) G_{l m}(Q)
$$

If the statistical weights are assumed to be constant, as in (Sacerdote \& Sansò, 1987), equation [3.6] becomes

$$
\begin{aligned}
& P_{2}^{-1} G_{l m}(Q)+\frac{p_{1}^{-1}}{4 \pi} \sum_{l^{\prime} m^{\prime}} \frac{\left(l^{\prime}+1\right)^{2}\left(l^{\prime}+2\right)^{2}}{\left(l^{\prime}-1\right)^{2} R_{1}^{2}}\left(\frac{R_{1}}{r_{Q}}\right)^{l^{\prime}+3} Y_{l^{\prime} m^{\prime}}\left(\sigma_{Q}\right) . \\
& \int_{S} d \sigma_{Q^{\prime}} \int_{R}^{R+\delta R} r_{Q^{\prime}}^{2} d r_{Q^{\prime}} G_{l m}\left(Q^{\prime}\right)\left(\frac{R_{1}}{r_{Q^{\prime}}}\right)^{l^{\prime}+3} Y_{l^{\prime} m^{\prime}}\left(\sigma_{Q^{\prime}}\right)= \\
& =\frac{P_{1}^{-1}}{4 \pi} \frac{(l+1)(l+2)}{(l-1)^{2}}\left(\frac{R_{T}}{r_{Q}}\right)^{l+3} Y_{l m}\left(\sigma_{Q}\right)
\end{aligned}
$$
form

Equation [3.8] implies $l=l^{\prime}, m=m^{\prime}$; moreover $G_{l m}(Q)$ must assume the

$$
G_{l m}(Q)=g_{l m} Y_{l m}\left(\sigma_{Q}\right)\left(\frac{R_{1}}{r_{Q}}\right)^{l+3}
$$

Consequently, performing the integration over $S_{1}$, one obtains

$g_{l m}=\frac{p_{2}}{4 \pi}\left[p_{1}(l-1)^{2}+p_{2}(l+1)^{2}(l+2)^{2} \int_{R}^{R+\delta R}\left(\frac{R_{1}}{r}\right)^{2 l+4} d r\right]^{-1}(l+1)(l+2)$

It is remarkable that, when $\delta R$ tends to 0 (i.e. if the shell where gradiometric measurements are performed is very thin with respect to the 
orbit radius, as in nearly circular orbits), this result agrees with the one obtained in (Sacerdote \& Sansò 1987) (see for example formula [2.33]).

In conclusion, no particular difficulty arises if "boundary" data are distributed in a volume and not on a surface. This peculiarity obviously is due to the fact that inaccuracies of the data are taken into account and represented stochastically.

\section{CONCLUSIONS}

It is clear that, as soon as the assumptions are slightly generalized with respect to the drastically simplifying ones of the previous papers, the task of analytically solving the problems and even of finding useful formulas for rough estimations of the results becomes out of reach. Therefore the investigations that have been carried out up to now have the main purpose to provide a theoretical basis for setting up numerical methods in order to deal with real data.

It can be predicted that, as it has occurred with the theory of classical boundary-value problems, the frame of overdetermined problems will prove more and more fitting to practical situations.

\section{References}

Magnus, W., Ober hetTinger, F. \& R. P. Soni (1966): Formulas and Theorems for the Special Functions of Mathematical Physics, 3.a ed., Springer-Verlag.

Mainville, A. (1986): The Altimetry-Gravimetry Problem using Orthonormal Base Functions, Rep. 373, Dept. of Geod. Sci. and Surv., OSU.

RuMMEL, R. \& P. TEUNISSEN (1987): Geodetic boundary-value problems and linear inference, Proc. Int. Symp. Figure and Dynamics of the Earth, Moon and Planets (P. Holota, ed.), Praha, Part I, pp. 227-264.

SACERDOTE, F. \& F. SANSO (1985a): The overdetermined boundary-value problems of physical geodesy, Man. Geod., 10, pp. 195-207.

SACERDOTE, F. \& F. SANSO (1985b): Esempi di problemi al contorno sopradeterminati della geodesia fisica, Atti del 4. Convegno annuale del GNGTS, Roma, pp. 10071014.

SACERDOTE, F. \& F. SANSO (1987): On the overdetermined gravimetry-gradiometry problem in physical geodesy,, Proc. Int. Symp. Figure and Dynamics of the Earth, Moon and Planets (P. Holota, ed.), Praha, Part I, pp. 265-276.

SANSO, F. (1988): The Wiener Integral and the overdetermined boundary-value problems of physical geodesy, Man. Geod., 13, 75-98.

Dipartimento di Matematica 